\title{
Serous and Mucinous Borderline Ovarian Tumours: Evaluation of Clinical Outcome and Risk Factors for Recurrence
}

\author{
Nesrine Tounsi ${ }^{1 *}$, Hatem Bouzaine', Raoudha Doghri², Hanen Bouazize ${ }^{1}$, Maher Slimane ${ }^{1}$, Jamel Ben Hassouna ${ }^{1}$ and Khaled Rahel' \\ ${ }^{1}$ Department of Surgery Carcinologique, Salah-Azaiez Institute, Boulevard, Tunis, Tunisia
}

${ }^{2}$ Department of Pathology, Salah-Azaiez Institute, Boulevard, Tunis, Tunisia

\begin{abstract}
Objective: Borderline Ovarian Tumors (BOTs) have a good prognosis; however, a few BOT patients experience the relapse of disease, either borderline or malignant. The aims of this study were to analyze the risk factors of relapse.

Methods: This is a retrospective study of 31 patients with confirmed BOTs treated in the Salah Azaiez Oncologic institute between 2005 and 2015.

Results: 31 cases were identified; median age was 43 years. Most of the patients $27(87 \%)$ demonstrated stage IA. 8 patients had laparoscopic surgery and they were all treated conservatively. Between 23 patients, which had laparotomy, only two cases underwent conservative surgery and the remaining patients had radical surgery. Five patients had recurrences $(16.13 \%)$. The relapse was occurred meanly 62.38 months after the primary surgery. Among the 5 recurrent cases, one cancerous transformation and 4 borderline recurrences were detected. 3 recurrences of 8 Laparoscopic surgery group and 2 recurrent cases of 23 laparotomic surgery groups were observed but the statistic result of the relation between laparoscopic approach and higher recurrence rate was not significant $(P=0.093)$. 3 recurrences of 10 conservative surgery group and 2 recurrent cases of 21 radical surgery groups were observed. However, it did not show significant difference $(P=0.175)$. Mean age of disease-recurrence group was 42.8 years whereas the one of non-recurrent group was 46.5 years $(P=0.65)$, age was not risk factor of disease recurrence.
\end{abstract}

Conclusion: Even if age, conservative treatment and laparoscopic technique does not reach the statistic significance as a risk factors for recurrence, we can have a definite conclusion. It is necessary to perform more randomized controlled trials to confirm such an assumption.

Keywords: Borderline ovarian tumour; Conservative surgery; Cystectomy; Recurrence

\section{Introduction}

Borderline ovarian tumors (BOTs) are an intermediate state of ovarian disease that does not specifically meet criteria for being either benign or malignant. The incidence rate of BOTs is 1.4-3.1 per 100,000 [1]. They are characterized by atypical epithelial proliferation without stromal invasion [2]. They exist in various subtypes, including serous, mucinous, seromucinous, clear cell, endometrioid and Brenner [3]. The first two subtypes account for approximately $95 \%$ of borderline tumors [4]. Even though most patients with BOT have a good prognosis, a minority will get a relapse, either borderline or malignant. The recurrence rate of BOTs has been reported to range from $5 \%$ to $33 \%$ in different published series [4-7]. The aims of this study were to analyze the risk factors of relapse and to compare the type of recurrence in patients with borderline tumors treated and followed up at a single institution, Salah Azaiez Oncologic institute.

\section{Material and Methods}

We performed a retrospective study of 55 patients with BOTs treated at Salah Azaiez institute between 2005 and 2015. Retrospective data were retrieved from hospital records and patient charts. Seven were excluded because of insufficient data, 3 because of concomitant malignancies ( 2 breast cancers and 1 gastric cancer) and 10 were eliminated because they had invasive implant or stromal microinvasion. A further 4 patients were eliminated from our study because of incomplete histological diagnoses of their recurrent tumors. Tumors of all clinical stages were reviewed and included in the study. Staff pathologists reviewed all cases and assisted in the tumor staging according to the International Federation of Gynecology and Obstetrics (FIGO) staging system. Operative reports were reviewed to confirm intraoperative findings at the time of initial surgery. Our statistical analysis therefore involved 31 patients. Information collected included patient age, parity, menopausal status, CA-125, tumor size, stage (International Federation of Gynecology and Obstetrics (FIGO), recurrence, malignant transformation, histological type on primary diagnosis and on relapse, site of recurrence, washing cytology and intra-operative rupture of tumor on primary surgery, surgical modality of primary and secondary surgery (laparoscopy vs. laparotomy), surgical category of primary and secondary surgery (conservative vs. comprehensive operation), operative extent of primary surgery (cystectomy vs. oophorectomy) and patient's status on last follow-up were obtained for analysis. The Chi square test or Fisher's test, when appropriate, were used to compare categorical variables. Univariate logistic analysis was performed to identify potential risk factors. Statistical significance was defined as $\mathrm{p}<0.05$.

\section{Surgical Procedures}

For patients who underwent surgery in our department, the

*Corresponding author: Nesrine Tounsi, Salah Azaiz Institute, Grombalia Nabeul, Tunisia, Tel: 99430944; E-mail: neserine.tounsi@gmail.com

Received September 10, 2017; Accepted October 11, 2017; Published October 16, 2017

Citation: Tounsi N, Bouzaine H, Doghri R, Slimane M, Hassouna JB, et al. (2017) Serous and Mucinous Borderline Ovarian Tumours: Evaluation of Clinical Outcome and Risk Factors for Recurrence. J Cancer Sci Ther 9: 709-712. doi:10.4172/19485956.1000495

Copyright: (C) 2017 Tounsi N, et al. This is an open-access article distributed under the terms of the Creative Commons Attribution License, which permits unrestricted use, distribution, and reproduction in any medium, provided the original author and source are credited. 
Citation: Tounsi N, Bouzaine H, Doghri R, Slimane M, Hassouna JB, et al. (2017) Serous and Mucinous Borderline Ovarian Tumours: Evaluation of Clinical Outcome and Risk Factors for Recurrence. J Cancer Sci Ther 9: 709-712. doi:10.4172/1948-5956.1000495

radicality of primary surgery was decided on the basis of the woman's age and her wish to preserve her fertility. As a general rule, in our department any surgical specimen is sent for frozen-section analysis. Radical surgery consisted of bilateral salpingo-oophorectomy (BSO) with or without the uterus. Conservative surgery was defined as sparing the uterus and at least one ovary. After successful pregnancies, conservative treatment was followed by Radical surgery. Staging was considered complete if it included, as a minimum, omentectomy with peritoneal biopsies. Patients were provided follow-up care every 6 months. More intensive closer follow-up was also recommended for patients receiving conservative treatment. Follow-up consisted of gynecological examination, tumor marker evaluation, and transvaginal ultrasound. This study received approval from our in-house institutional review board.

\section{Results}

Our study included 31 patients, Median age of the patients at diagnosis was 43 years old (21-87 years) and 15 (48.38\%) patients were below 40 years-old at their first diagnosis of BOTs. Seven $(22.58 \%)$ patients were nulliparous.

Most presented with the following preoperative symptoms: abdominal swelling, pain, and abnormal uterine bleeding. Preoperative ultrasound examinations suggested a suspicious mass in most patients (86.6\%). However, for the other patients, the ultrasound examinations indicated a unilocular cyst with no signs of malignancy.

The clinical and pathologic characteristics of the entire cohort are presented in Table 1.

Most of the patients 27(87\%) demonstrated stage IA, two patients presented with stage IB, one woman with stage IIB which involved the pouch of Douglas with non-invasive implants and one woman presented stage IIIB which involved bilateral salpinges, omentum and peritoneum with non-invasive implants.

The dominant histopathological subtypes were serous $(21$ cases; $67.7 \%$ ) and mucinous (9 cases; $29 \%$ ) patients and one patient with seromucinous type. Eight (25.8\%) cases were treated initially by laparoscopic approach, and $23(74.2 \%)$ by laparotomy (Figure 1 ). All laparoscopic operations were conservative. Among patients who received conservative surgery, only one cases had cystectomy and the others undertook unilateral oophorectomy with omentectomie and appendectomy. Among 23 patients, which had laparotomy, 2 cases underwent conservative surgery. 21 (91.30\%) patients received radical surgery of BOTs with bilateral oophorectomy, omentectomie, appendectomy and with or without hysterectomy. In fact, There were some debatable cases, two patients underwent BSO without

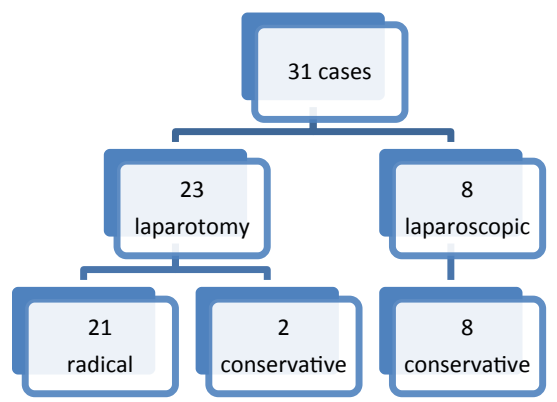

Figure 1: Diagram of the borderline ovarian tumor patients in terms of the surgical approach.

\begin{tabular}{|c|c|c|c|c|}
\hline Variables & $\begin{array}{c}\text { Patients } \\
\text { characteristics }\end{array}$ & Recurrence & $\begin{array}{c}\text { Died of } \\
\text { disease }\end{array}$ & $\begin{array}{c}\text { Died of } \\
\text { ICD }\end{array}$ \\
\hline Number of patients & 31 & 5 & 1 & 1 \\
\hline Age & 0 & 0 & 0 & 0 \\
\hline$<20$ & $25(80.6 \%)$ & 4 & 1 & 0 \\
\hline $20-60$ & $6(19.4 \%)$ & 1 & 0 & 1 \\
\hline$>60$ & &
\end{tabular}

\section{Parity}

\begin{tabular}{|c|c|c|c|c|}
\hline Nulliparous & 7 & 2 & 1 & 0 \\
\hline Multiparous & 24 & 3 & 0 & 1 \\
\hline Histological type & 9 & 1 & 0 & 1 \\
\hline Mucinous & 21 & 4 & 1 & 0 \\
\hline Serous & 1 & 0 & 0 & 0 \\
\hline Mixed & &
\end{tabular}

Stage

\begin{tabular}{|c|c|c|c|c|}
\hline IA & 27 & 4 & 1 & 1 \\
\hline IB & 2 & 0 & 0 & 0 \\
\hline IC & 0 & 0 & 0 & 0 \\
\hline II & 1 & 0 & 0 & 0 \\
\hline IIIA & 0 & 0 & 0 & 0 \\
\hline IIIB & 1 & 1 & 0 & 0 \\
\hline Surgical procedure & \multicolumn{1}{|l|}{} \\
\hline Radical & 21 & 2 & 0 & 1 \\
\hline Conservative & 10 & 3 & 1 & 0 \\
\hline
\end{tabular}

\section{Types of surgery}

LS conservative

LT conservative

$\mathrm{LT}$ radical

\begin{tabular}{|c|l|l|l|}
\hline 8 & 3 & 1 & 0 \\
\hline 2 & 0 & 0 & 0 \\
\hline 21 & 2 & 0 & 1 \\
\hline
\end{tabular}

Adjuvant chemotherapy

\begin{tabular}{|c|c|c|c|c|}
\hline Yes & 2 & 2 & 1 & 0 \\
\hline No & 26 & 3 & 0 & 0 \\
\hline
\end{tabular}

ICD: Inter Current Disease; LS: Laparoscopic; LT: Laparotomy

Table 1: The clinical and pathologic characteristics.

\begin{tabular}{|c|c|c|}
\hline \multicolumn{1}{|c|}{ Factors } & Recurrence & No recurrence \\
\hline Mean age (years) & 42.8 & 46.5 \\
\hline Stage, $\mathbf{n}$ (\%) & 4 & 25 \\
\hline I II & 1 & 1 \\
\hline Histology & \multicolumn{2}{|c|}{} \\
\hline Serous & 4 & 21 \\
\hline Mucinous & 1 & 9 \\
\hline Mixed & 0 & 1 \\
\hline IECA & 1 & 0 \\
\hline Micropapillary & 1 & 0 \\
\hline Stromal Microinvasion & 0 & 0 \\
\hline Invasive implants & 0 & 0 \\
\hline Non-invasive implants & 2 & 1 \\
\hline
\end{tabular}

Table 2: Characteristics of patients with recurrence of disease.

hysterctomie; one because she had decompensated cirrhosis with a high risk of anesthesia, and other patient whose age was 21-yearold at primary diagnosis of bilateral BOT and she had had bilateral salpingo-oophorectomie because she wanted to preserve her uterus when discussing about the management of her BOT with the doctor. Finally, she had BSO with remaining her uterus. During the mean follow-up time of (62.38) months (range 12 to 132 months). Five patients have encountered recurrences (16.13\%). (Tables 2 and 3 ) shows characteristics of patients with recurrence of disease. In 2 cases relapses were intrapelvic: one was ipsilateral, one contralateral ovary and for the other were in form of pelvic implants. These patients were managed with 


\begin{tabular}{|c|c|c|c|c|c|c|c|c|}
\hline Age (year) & $\begin{array}{c}\text { Pathology on } \\
\text { primary diagnostic }\end{array}$ & stage & Initial surgery & $\begin{array}{c}\text { Time to recurre } \\
\text { (months) }\end{array}$ & Site of recurrene & $\begin{array}{l}\text { Treatment after } \\
\text { recurrent (months) }\end{array}$ & $\begin{array}{l}\text { Histolog Pathology on } \\
\text { recurrences reccure }\end{array}$ & status \\
\hline 36 & SBT, non invasive & IA & Conervative (LS) & 120 & peritoneal implant & Radical Surgery +CT & $\begin{array}{c}\text { Serousa } \\
\text { Adénocarcinoma }\end{array}$ & DOD \\
\hline 87 & MBT, non invasive & IA & Radical (LT) & 36 & peritoneal & Surgery & MBT, non invasive & Died of ICD \\
\hline 21 & $\begin{array}{l}\text { SBT, non invasive } \\
\text { MPs }\end{array}$ & III & Radical (LT) & 122 & peritoneal implant & Surgery $+\mathrm{CT}$ & SBT, non invasive & NED \\
\hline 32 & SBT, non invasive & IA & Conservative (LS) & 60 & Same ovary & Surgery & SBT, non invasive & NED \\
\hline 38 & SBT, non invasive & IA & Conservative (LS) & 24 & contralateral ovary & conservative Surgery & SBT, non invasive & NED \\
\hline
\end{tabular}

NED: No Evidence of Disease; SBT: Serous Borderline Tumour, MBT: Mucinous Borderline Tumour; IECA: Intraepithelial Carcinoma; Mps: Micropapillary Patterns; CT= Cystectomy, DOD: Died of Disease, ICD: Intercurrent Disease; LS: Laparoscopie; LT: Laparotomy

Table 3: Patients with recurrence of disease.

a second surgical intervention, only one was conservative (1/5). Among patients with recurrence of disease, four revealed recurrent borderline tumors and one case developed invasive carcinoma. Invasive relapses occurred in one patient (stage IA) who had received conservative surgery as treatment of choice based on the clinical presentation. The relapse was occurred 120 months. Relapses were peritoneal carcinosis localized to the pelvis and she received chemotherapy, 24 months after invasive relapses, the patient develops a neoplastic pleurisy and she died a short time after. Relapse as a borderline tumor occurred predominantly in 4 patients who had initially received conservative treatment in 3 and radical in one patient. The mean age of this cohort of patients was 44.67 years, with a median time to relapse of 72.4 months (range 24 to 122 months). Among 8 patients who had laparoscopic conservative surgery group, 3 cases had recurrence. In our data shows, that age was not risk factor of disease recurrence. Mean age of diseaserecurrence group was 42.8 years old whereas the one of non-recurrent group was 46.5 years old $(\mathrm{P}=0.65)$. Moreover, laparoscopic approach was not related to higher recurrence rate $(\mathrm{P}=0.093) .3$ recurrences of 10 conservative surgery group and 2 recurrence cases of 21 radical surgery group were observed. However, it did not Show significant difference $(\mathrm{P}=0.175) .4$ recurrence of 9 serous borderline tumor was occurred and one recurrence of 21 mucinous borderline tumor was occurred, no significant difference $(\mathrm{p}=0.472)$. From 31 patients, two patients were died: the first patient has 87-year-old died from septic shock secondary to pneumonia few days after her operation for recurrent disease and the second died from progression of her recurrent disease. The remaining patients are still alive in a complete remission status according to the last check up.

\section{Discussion}

BOTs are classified as a separate entity within ovarian malignancies because of their atypical properties [8]. BOT present tow dilemma: frequently, it reaches a younger fertile woman often wishing to attain a viable pregnancy and, it presents a height rates of recurrence range from $5 \%$ to $33 \%$ especially when treatment of choice was conservative surgery [9]. Hopefully the majority of recurrences of borderline tumors may be salvaged surgically but sometimes it transforms to ovarian malignancy [9]. The vast majority of clinicians consider radical treatment (total abdominal hysterectomy with bilateral salpingooophorectomy and omentectomy) as the best alternative to ensure lower risk of recurrence range from $2.5 \%$ to $5.7 \%$ [10]. In other hand, young women wishing fertility make clinicians to hesitate to follow up the standard guidelines. As this, many clinicians believe that a conservative approach is therefore reasonable, followed in some cases by definitive surgery after successful pregnancies [11]. Conservative surgery is a subject of debate because is associated with a higher incidence of recurrences. When only cystectomy was performed, the risk of recurrence was between 12 and $58 \%$. However, such a risk is lower $(0 \%$ to $20 \%$ ) when salpingo oophorectomy was performed [6]. In early stage
I, conservative treatment is strongly recommended [12]. In the study of Shih et al. [13] risk of recurrence was higher in patients who undergo conservative surgery compared with those patients who had radical surgery. Nevertheless, they thought that conservative fertility-sparing surgery remains a reasonable option in the management of BOT. Uzan et al. [12] reported a series of 41 patients treated conservatively for an advanced-stage serous borderline ovarian tumor. Their results show that conservative surgery can be safely proposed to patients with no invasive peritoneal implants who are carefully followed up because their prognosis remains good if implants are totally resected [14]. In the series reported by Zanetta et al. [6], disease-free survival of patients with stage II and III lesions was similar in conservatively or radically treated patients. In advanced stage II and III, few study evaluated the benefit of conservative treatment because the majority of recurrences occur in patients who present with advanced disease when compared to early disease [15-17] and especially for those with invasive implant. In brief, we believe that conservative attitude is a reasonable approach after adequate and complete staging consisting of careful inspection of the peritoneal cavity with multiple biopsies, omentectomy and appendectomy, followed in some cases by definitive surgery after successful pregnancies. Moreover, the overall survival for patients who undergo conservative treatment was not affected because most replaces may be salvaged by surgery $[11,14]$. In our study, 3 recurrences of 10 conservative surgery group and 2 recurrent cases of 21 comprehensive surgery groups were observed. However, it did not Show significant difference $(\mathrm{P}=0.175)$. While lymphadenectomy is part of surgical staging in ovarian cancer, it has not been shown to be prognostically significant in BOT [15]. In many study, lymph node dissection or nodal involvement has no impact on recurrence or survival $[13,16,17]$. This finding provides further evidence that routine lymphadenectomy may not be necessary with BOT. In our study, none patients undergoing lymph node dissection. The choice between lapartomy vs laparoscopy is subject of debate. Some data suggest that overall recurrence is lower with complete surgical staging at the time of presentation. Therefore, most gynecologist oncologists still consider midline laparotomy as the gold standard approach because we could do complete surgical staging [18]. However In terms of surgical outcomes, laparoscopic approach provides some benefits: Small incisions, quicker recovery time and lower risk of adhesions. Favorable oncological results and subsequent pregnancies have been reported after conservative management $[19,20]$. In the study of $\mathrm{Oh}$ et al. laparoscopic approach was related to higher recurrence rate and lower DFS [8]. This is probably due to the higher risk of intraoperative tumor rupture or incomplete staging [8]. Our data show no significant difference $(\mathrm{P}=0.175)$ between laparoscopic approach surgery group and open surgery group. But we can draw a significant conclusion because there were none who had laparoscopic comprehensive operation. Several factors predictive of replace were reported in different studies: Conservative surgery, cyst rupture, tumor size, FIGO stage, micro papillary pattern, micro invasion and peritoneal 
implant [21,22]. Vasconcelos et al. recommended that patient with established risk factors for recurrence such as residual disease after up-front surgery and the presence of invasive implants, should be particularly advised to undergo more aggressive surgical procedures, while patients with micropapillary pattern and stromal micro invasion in serous BOT and intra-epithelial carcinoma in mucinous BOT should be made careful to these apparent risk factors and act accordingly [9]. In the largest series reported to date, they identified that the risk factor of recurrence was related to young age, tumor bilaterality and the use of a cystectomy suggesting that management of fertility preservation (particularly in very young patients) should be associated with a meticulously conducted follow-up [11]. In our data, we reported only one case (stage III), who had borderline recurrence after 122 months from radical surgery. However, invasive relapses occurred in one patient (stage IA) who received conservative surgery as treatment of choice based on the clinical presentation. The relapse was occurred 120 months. Relapses were peritoneal carcinosis localized to the pelvis and she received second surgery for invasive implant followed by chemotherapies, 24 months after invasive relapses, the patient develops a neoplastic pleurisy and she died a short time after. The unexpected progress of our patient, allows us to ask about the factors related of replaces. Long-term follow-up, for 10 years or more after intervention for BOTs should be performed [8]. Similarly, Silva et al. [23] suggested that patients who were treated by BOTs should be followed for a minimum of 10 years to evaluate for recurrence and for 20 years to evaluate for survival. In the study of Shih et al. [13], most of relapses occur during the first 2 years after surgery, are easily detected with transvaginal ultrasound, and can be successfully managed surgically. Patients with borderline replace, have a very good prognosis even if the secondary surgery is conservative. Conversely, invasive relapses are uncommon but connected with poor prognosis. These relapses can arise after many years and their diagnosis may not be straightforward. These patients, at the time of relapse, will have advanced disease with significant pelvic spread and sometimes involvement of metastases to lymph nodes. Treatment comprises extensive tumor and pelvic organ resection and multiple courses of adjuvant chemotherapy, which are often unsuccessful [18]. Most study observed that was a small difference in the rate of recurrence between serous and mucinous tumors [24]. Our results didn't show significant difference $(p=0.472)$ between mucinous or serous replaces.

\section{Conclusion}

Our data show that the risk of progression to invasive carcinoma does exist but is low. Most patients was stage IA it according with literature. In this study, total recurrence rate of BOTs was $16.13 \%$ is higher comparing with other studies. Our study has several limitations, the most relevant are relatively small sample size, and none patient had laparoscopic comprehensive operation. Finally, even if age, conservative treatment and laparoscopic technique do not reach the statistic significance as risk factors for recurrence in our study, this factor seems to influence the outcome. It is necessary to perform more randomized controlled trials to confirm such an assumption.

\section{References}

1. Morris CR, Liu L, Rodriguez AO, Cress RD, Snipes K (2013) Epidemiologic features of borderline ovarian tumors in California: A population-based study. Cancer Causes Control 24: 665-674.

2. Acs $G$ (2005) Serous and mucinous borderline (low malignant potential) tumors of the ovary. Am J Clin Pathol 123: 13Y57.

3. Song T, Choi CH, Lee YY, Kim TJ, Lee JW, et al. (2013) Endocervical-like versus intestinal-type mucinous borderline ovarian tumors: A large retrospective series focusing on the clinicopathologic characteristics. Gynecol Obstet Invest 76: 241-247.

4. Lalwani N, Shanbhogue AKP, Vikram R, Nagar A, Jagirdar J, et al. (2010) Current update on borderline ovarian neoplasms. AJR Am J Roentgenol 194: 330-336.

5. Lenhard MS, Mitterer S, Kumper C, Stieber P, Mayr D, et al. (2009) Long-term follow-up after ovarian borderline tumor: Relapse and survival in a large patient cohort. Eur J Obstet Gynecol Reprod Biol 145: 189-194.

6. Zanetta G, Rota S, Chiari S, Bonazzi C, Bratina G, et al. (2001) Behavior of borderline tumors with particular interest to persistence, recurrence, and progression to invasive carcinoma: a prospective study. J Clin Oncol 15: 192658-192664.

7. Du Bois A, Ewald-Riegler N, De Gregorio N, Reuss A, Mahner S, et al. (2013) Borderline tumours of the ovary: A cohort study of the Arbeitsgmeinschaft Gynakologische Onkologie (AGO) Study Group. Eur J Cancer. 49:1905-1914.

8. Oh S, Kim R, Lee YK, Kim JW, Park NH, et al. (2015) Clinicopathological aspects of patients with recurrence of borderline ovarian tumors. Obstet Gynecol Sci 58: 98-105.

9. Vasconcelos I, Darb-Esfahani S, Sehouli J (2016) Serous and mucinous borderline ovarian tumours: Differences in clinical presentation, high-risk histopathological features, and lethal recurrence rates. BJOG 123: 498-508.

10. Cadron I, Amant F, Van Gorp T, Neven P, Leunen K, et al. (2006) The management of borderline tumours of the ovary. Curr Opin Oncol 18: 488-493.

11. Suh-Burgmann $E$ (2006) Long-term outcomes following conservative surgery for borderline tumor of the ovary: A large population-based study. Gynecol Oncol 103: 841-847.

12. Uzan C, Zanini-Grandon AS, Bentivegna E, Gouy S, Leary A, et al. (2015) Outcome of patients with advanced-stage borderline ovarian tumors after first peritoneal noninvasive recurrence: Impact on further management Int $\mathrm{J}$ Gynecol Cancer 25: 830-836.

13. Shih KK, Zhou Q, Huh J, Morgan JC, lasonos A, et al. (2011) Risk factors for recurrence of ovarian borderline tumors. Gynecol Oncol 120: 480-484

14. Julian CG, Woodruff JD (1972) The biologic behavior of low grade papillary serous carcinoma of the ovary. Obstet Gynecol 40: 860-867.

15. Winter WE $3^{\text {rd }}$ (2002) Surgical staging in patients with ovarian tumors of low malignant potential. Obstet Gynecol 100: 671-676.

16. Barakat RR (1994) Borderline tumors of the ovary. Obstet Gynecol Clin North Am 21: 93-105.

17. Kaern J, Trope CG, Kristensen GB, Abeler VM, Pettersen EM (1993) DNA ploidy; the most important prognostic factor in patients with borderline tumors of the ovary. Int J Gynecol Cancer 3: 349-358

18. Sobiczewski P, Kupryjanczyk J, Michalski W, Śpiewankiewicz B (2016) The evaluation of risk factors associated with relapse and recurrence of borderline ovarian tumors with long-term follow-up. Int J Gynecol Cancer 26: 1053-1061.

19. Laurent I, Uzan C, Gouy S, Pautier P, Duvillard P, et al. (2008) Results after conservative treatment of serous borderline tumors of the ovary with a micropapillary pattern. Ann Surg Oncol 15: 3561-3566.

20. Leary A, Petrella MC, Pautier P, Duvillard P, Uzan C, et al. (2014) Adjuvant platinum-based chemotherapy for borderline serous ovarian tumors with invasive implants. Gynecol Oncol 132: 23-27.

21. Ren J, Peng Z, Zang K (2008) A clinicopathologic multivariate analysis affecting recurrence of borderline ovarian tumors. Gynecol Oncol. 110: 162-167.

22. Kristensen GS, Schledermann D, Mogensen O (2014) The value of random biopsies, omentectomy and hysterectomy in operations for borderline ovariantumors. Int J Gynecol Cancer 24: 874Y879.

23. Silva EG, Gershenson DM, Malpica A, Deavers M (2006) The recurrence and the overall survival rates of ovarian serous borderline neoplasms with noninvasive implants is time dependent. Am J Surg Pathol 30: 1367-1371.

24. Black JD, Altwerger GH, Ratner E, Lu L, Silasi DA (2015) Management of borderline ovarian tumors based on patient and tumor characteristics. Gynecol Obstet Invest. 